\title{
Tätigkeitsbericht des Phonogrammarchivs für das Jahr 2013
}

\section{Forschung}

Im Forschungsschwerpunkt „Researching and documenting endangered languages of Kinnaur" führte Christian Huber seine Erforschung des Shumcho, einer bedrohten und bislang nicht dokumentierten tibeto-birmanischen Sprache des District Kinnaur (Himachal Pradesh, Indien), weiter. Die 2013 durchgeführten Forschungen machten sich in der Feldforschung auch moderne Kommunikationstechnologien zunutze und brachten weitere Erkenntnisse vor allem zu den Modalverben und zur Auxiliarselektion. Die Ergebnisse der neueren Forschung zur Verbalmorphologie wurden in Vorträgen (z.B. an der Universität Bern) und einer ausführlichen Publikation (s. Publikationsliste) der wissenschaftlichen Öffentlichkeit zugänglich gemacht.

Hedwig Köb setzte ihr stadtethnologisches Langzeitprojekt „Dokumentation kultureller Aktivitäten westafrikanischer Zuwanderer in Wien" mit der Videodokumentation des an verschiedenen Plätzen Wiens und im Weltmuseum stattfindenden "Nigeria Cultural Festival“ („Nigerian week“) fort, und Katharina Thenius-Wilscher arbeitete weiterhin an ihrer Re-Study zur Volks- und Popularmusikpraxis in Österreich (audiovisuelle Dokumentation aktueller Ereignisse mit Musik im Industrieviertel/NÖ).

Aus dem Forschungsschwerpunkt "Mechanische Musikinstrumente", der von Helmut Kowar verfolgt wird, wurden die Ergebnisse der virtuellen Rekonstruktion, die Tondokumentation und die Untersuchungen zur Musik der Renaissanceautomaten des Kunsthistorischen Museums (Trompeterautomat, 1582; Automat in Form eines Schiffes, 1585; Bacchuswagen, ca. 1602-1606; Orgelwerke des Walbaum-Schrankes, um 1620/25, und des Triumphwagens mit Minerva, 1625-30) publiziert (s. Publikationsliste).

Die MitarbeiterInnen der Abteilung „R.M. Brandl-Collection“ am Phonogrammarchiv widmeten sich zum einen mit zahlreichen Videoaufnahmen der Weiterführung des 1973 begonnenen Langzeit-Dokumentationsprojekts „Wiener Volkssänger und traditionelle Wiener Volksmusik“, zum anderen aber auch der laufenden Archivierung und Aufarbeitung der Bestände an Aufnahmen der chinesischen Kunqu-Oper.

Jürgen Schöpf organisierte gemeinsam mit Mélanie Vandenhelsken (Institut für Sozialanthropologie, ÖAW), Shahnaz Leblhuber (Institut für Südasien-, Tibet- und Buddhismuskunde, Universität Wien) und Bianca Son (SOAS, London) die internationale Konferenz „Negotiating Ethnicity: Politics and Display of Cultural Identities in Northeast India" an der Universität Wien 
(mit Unterstützung der Botschaft der Republik Indien). 43 Konferenzbeiträge, darunter zwei Fotoausstellungen und drei Filmvorführungen, setzten einen Schwerpunkt in der sich gerade rasch entwickelnden Forschung über diese dynamische Region Asiens. Etwa die Hälfte der Vortragenden kam aus Indien, darunter sehr viele junge Forscher, deren Teilnahme nur durch finanzielle Unterstützung aus Mitteln der ÖAW möglich war.

Im Auftrag des Burgenländischen Volksliedwerkes gab Christiane Fennesz-Juhasz gemeinsam mit Emmerich Gärtner-Horvath (Verein RomaService, Kleinbachselten) Dschila le Romendar andar o Burgenland heraus. Konzipiert für den praktischen Gebrauch, enthält das zweisprachige Liederbuch Noten und Texte von traditionellen und neueren Liedern der Burgenland-Roma sowie der Lovara (bis zum Zweiten Weltkrieg ebenfalls in diesem Bundesland heimisch), darüber hinaus aber auch Kommentare zu deren Herkunft, Kontext und Ausführung sowie deutsche Übersetzungen der Romani-Texte und detaillierte Quellenangaben. Für die Publikation wurde einerseits bereits veröffentlichtes Material neu aufbereitet, andererseits waren 18 der insgesamt 30 Lieder erst zu transkribieren, wobei hier z.T. auf Bestände des Phonogrammarchivs zurückgegriffen werden konnte.

Der von Christian Liebl zusammen mit Michael Studemund-Halévy (IGDJ - Institut für die Geschichte der deutschen Juden, Hamburg) und Ivana Vučina Simović (Universität Kragujevac, Serbien) herausgegebene Sammelband Sefarad an der Donau, der teilweise auf den Beiträgen zu der vom Phonogrammarchiv mitorganisierten internationalen Konferenz 2011 an der ÖAW basiert, konnte Ende Mai in der ÖAW präsentiert werden.

Die international herausragende Stellung des Phonogrammarchivs im Bereich der technisch-konservatorischen Langzeitsicherung von audiovisuellen Datenträgern konnte durch die rezenten Forschungen im Berichtsjahr ausgebaut werden. Nachdem 2011 eine Methode zur Rekonditionierung extrem gealterter und völlig unspielbarer Tonbänder auf Cellulose-Acetatbasis zum Patent angemeldet worden war, wurde das Verfahren - die Patenterteilung ist noch ausständig - nun offiziell in Europa, USA, Kanada, Indien und weiteren Ländern Eurasiens angemeldet. 2013 wurde in einer Vorstudie die Anwendbarkeit der Methode auf gealterte Filmmaterialien ausgedehnt und geprüft, was durch die Gewährung eines Innovationsschecks der FFG ermöglicht wurde. Die Methode traf auch international bereits auf höchstes Interesse, kann derzeit aufgrund fehlender Mittel jedoch nicht in gewünschtem Maße weiterentwickelt werden. Die Zusammenarbeit mit einem der größten kommerziellen Archive weltweit wurde angebahnt.

Die Forschungen zur Extraktion des Vormagnetisierungssignals zur Korrektur von Gleichlaufschwankungen bei analogen Tonbandaufnahmen wurde insofern für Spezialfälle in den internen Archivworkflow als Prototyp 
eingebaut, als sie bei einzelnen Bändern aus dem EU-TEMPUS-Projekt "InMuSWB" (s. 5.) versuchsweise angewendet werden konnte.

\section{Technische Entwicklung im audiovisuellen Bereich}

\subsection{Beauftragte Digitalisierungsprojekte}

Die Sicherung und Digitalisierung der Tonbandsammlung des Oberösterreichischen Landesarchivs wurde im Frühjahr 2014 zur Gänze abgeschlossen. Es handelt sich dabei um 96 Tonbänder mit Aufnahmen von Landtagssitzungen.

Von Jänner bis März 2013 erfolgte eine Beratung zur Lagerung und Konservierung der Decelith-Platten des Universalmuseums Joanneum - Multimediale Sammlungen. Im Februar 2013 übernahm das Phonogrammarchiv als Auftragsarbeit die Digitalisierung eines Konvoluts von ca. 60 Schellackplatten aus dem Privatarchiv Maximilian Zundel. Die interessante Sammlung entstand während einer Reise durch Europa und Asien; der Vater des Sammlungsinhabers führte als Testfahrer für Mercedes Unimog in den frühen 1950er-Jahren diese Reise durch, die den Verlauf musikalisch widerspiegelt. Ebenfalls im Frühjahr 2013 erfolgte die Übertragung mehrerer Schellackplatten für die „Association for preservation and provision of Argentine Tango". Daraufhin erfolgte eine umfangreiche Beratung zur Digitalisierung der Schallplattenbestände.

Im Herbst 2013 wurde mit den Digitalisierungsarbeiten für das Projekt „Das Lautdenkmal reichsdeutscher Mundarten zur Zeit Adolf Hitlers Rekonstruktion, Edition, Analyse" (Christoph Purschke, Deutscher Sprachatlas Marburg) begonnen. Die Arbeiten an den 106 massiven Schellackplatten aus dem Bestand des Bayerischen Wörterbuchs und der Forschungsgruppe DINAMLEX am ICLTT sowie an den 23 Tonbändern (Masterkopien von den Muttermatrizen) aus dem Bestand des Forschungszentrums Deutscher Sprachatlas Marburg waren zum Ende des Berichtsjahres bereits zu mehr als zwei Drittel fertiggestellt.

2.2 Videographie, technische Sicherung von Videobeständen, Netzwerk

Die parallel zu den Routinearbeiten laufende Migration der Videobestände wurde mit drei Projekten fortgeführt (Kowar: Musik der Religionen 1996-1998; ÖVLW: Feldforschung Böhmerwald 2003; Pesendorfer: USA, März 2005). Die Migration der Videobestände wurde bewusst ohne jede Automatik und Schritt für Schritt mit Unterstützung einer Archivarin vorgenommen. Da es sich um die erste Migration handelt und der Workflow zu Beginn der Videoarchivierung 2002 mehrfach modifiziert wurde, um einerseits eine möglichst weitgehende Anpassung an die bewährten 
Arbeitsschritte der Audioarchivierung zu garantieren und andererseits dem technisch unterschiedlichen Quellmaterial gerecht zu werden, schien im derzeitigen Stadium - im Gegensatz zur zukünftigen 2. Migration - eine Automatisierung nicht sinnvoll.

Hedwig Köb führte ihre Arbeiten zur Optimierung des Workflows für die Videoarchivierung fort.

\section{Forschungsdatensammlung}

\subsection{Archivierung, Erschließung, Bild- und Tondokumentation}

Christian Huber schloss u.a. die Archivierung der Aufnahmen aus Spiti von Veronika Hein (2009) und der Aufnahmen von Beáta Wagner-Nagy aus Tajmyr (2008) ab. Hedwig Köb beendete die Archivierung der Feldaufnahmen "Kenga-Erzählungen, Tschad 1996" aus der Sammlung Peter Fuchs sowie der Aufnahmen der Gespräche mit emer. Univ.-Prof. Dr. Peter Fuchs zur Übernahme seiner Sammlung. Die Archivierung des zweiten Teils der Sammlung „Alfred Jilka, Vogelstimmen (1964-1983)“ wurde abgeschlossen (K. Thenius-Wilscher). Der Gesamtumfang der Sammlung Jilka im Phonogrammarchiv beträgt nun 143 Einheiten im Ausmaß von 36 Stunden. Ebenfalls abgeschlossen wurde die Archivierung der Audioaufnahmen historischer Musikautomaten aus den Jahren 2011-2013 (H. Kowar).

Die Digitalisierung der Aufnahmen von Karoly Gaál aus den Jahren 1961 bis 1973 (Teilbestand der Tonbandsammlung) wurde fertiggestellt und die Originalbänder dem Savaria Múzeum in Szombathely wieder zurückgebracht. Die arbeitsintensive "Re-Archivierung" (Erstellung der einzelnen Archiv-Wavefiles analog zu den ursprünglich auf Band archivierten Beständen und Zuordnung zur Archiv-Datenbank), die teilweise - wegen fehlender bzw. neu bespielter Originalbänder - unter Heranziehung digitalisierter Archivbandaufnahmen erfolgen musste, wird erst im Frühjahr 2014 fertiggestellt werden (C. Fennesz-Juhasz, N. Wallaszkovits, M. Födisch).

Mit der Übertragung von 38 Originalbändern (mono Halbspur) der Aufnahmen von Hermann Markus Preßl in Afghanistan 1967-1971 (B 1300113419, B 14691-14798) wurde ein weiterer Teilbestand der Tonbandsammlung digitalisiert; hierbei erfolgte ebenfalls zur Kontrolle die parallele Digitalisierung der entsprechenden Archivbänder. Die Segmentierung der Wavefiles und ihre Zuordnung in der Archiv-Datenbank ist für das Folgejahr geplant. Die Arbeiten wurden, mit Supervision durch die Arbeitsgruppenleiterinnen, bis Ende Juli vom Trainee Fíacha O'Dubhda und ab Herbst von der Fulbright-Stipendiatin Rachel Mandell, MA, durchgeführt. 
3.2. Datenbank, Online-Katalog und Website

In der Dokumentationsdatenbank wurde die Verbesserung bzw. Ergänzung der Metadaten der historischen, bereits in der CD-Edition erschienenen Aufnahmen sowie die Verknüpfung der entsprechenden Wave-Files mit den Datenbankeinträgen abgeschlossen; darüber hinaus wurden 20-Sekunden-Ausschnitte sämtlicher dieser Aufnahmen im Online-Katalog verfügbar gemacht und die Einträge mit direkten Links zu den betreffenden CDs auf der Website des ÖAW-Verlags ergänzt.

Die 2012 konzipierte Beta-Version der „Feld“-Datenbank wurde von zwei mit dem Phonogrammarchiv kooperierenden Feldforscherinnen getestet, wobei eine von innen ihre Dokumentation bereits während der Feldforschung (offline) eingegeben hat.

Das Phonogrammarchiv nahm den diesjährigen UNESCO Welttag des audiovisuellen Erbes am 27. Oktober zum Anlass, um erstmals im Rahmen einer Web-Ausstellung („HINeinSEHEN - HERausHÖREN: Ton- und Videodokumente aus dem Phonogrammarchiv“) Einblicke in die Vielfalt und Einzigartigkeit seiner Bestände zu geben (Konzept und Redaktion: Christian Liebl, Katharina Thenius-Wilscher; technische Realisierung: Johannes Spitzbart, Franz Pavuza, Michael Risnyovszky).

\section{Edition „Gesamtausgabe der Historischen Bestände 1899-1950“}

Als Serie 13 wurden das Manuskript und die Master-CD zu Recordings in Egypt (Junker 1911) and the Archive (Stigler 1912-1913) dem Publikationskomitee der ÖAW zur Begutachtung vorgelegt und einstimmig zum Druck empfohlen.

Durch eine großzügige finanzielle Unterstützung seitens der Cassa di Risparmio (Ravenna) konnten die Arbeiten zur Serie 14 Friedrich Schürr's Recordings from Romagna (1914) vorangetrieben und Manuskript samt Master-CD im Februar 2014 zur Begutachtung eingereicht werden.

Auch die Serie 15 "Indian Recordings" wurde weiter verfolgt. Letztlich wurde entschieden, diese Serie in zwei Teilserien herauszugeben: als Serie 15/1 (die Aufnahmen von Felix Exner aus den Jahren 1904/5 sowie die Archivaufnahmen von 1918) und als Serie 15/2 (die tamilischen Aufnahmen von Hilko W. Schomerus).

Für die Serie 16 „Recordings from the Caucasian Region 1909 and 1915/16" wurden vor allem die Aufnahmen aus den russischen Kriegsgefangenenlagern von 1915/16 (Aufnahmen von Pöch und Lach) transkribiert und kommentiert.

Für die weiteren ca. 200 Aufnahmen aus den Kriegsgefangenenlagern des Ersten Weltkriegs wurde das Projekt „,'Displaced Voices' - Tondokumente 
aus Kriegsgefangenenlagern im Ersten Weltkrieg" beim Jubiläumsfonds der Österreichischen Nationalbank eingereicht und Ende Dezember bewilligt. Somit ist es möglich, Frau Mag. Dr. Ulla Remmer ab 1. Februar 2014 halbtags für dieses Projekt anzustellen und außerdem für Spezialfragen Werkverträge zu vergeben.

\section{Kooperationen}

Das Projekt „European Acoustic Heritage“ (2011-2013) wurde mit Aktivitäten in Grenoble, Volos, Brüssel und mehreren Veranstaltungen in Spanien (Santiago de Compostela, Vigo und Pontevedra) erfolgreich abgeschlossen. Jürgen Schöpf trug als Projektmanager für das Phonogrammarchiv zu mehreren dieser Veranstaltungen die Erfahrungen mit Surround-Aufnahmesystemen unter Feldforschungsbedingungen sowie die Ergebnisse einer ReStudy des Projektes „Umweltgeräusche der Stadt Wien (1980-1983)“ bei. Außerdem gab Jürgen Schöpf eine Doppel-DVD mit dem Projekttitel heraus (technische Realisierung: Johannes Spitzbart).

Im 2011 angelaufenen EU-Projekt "Introducing interdisciplinarity in music studies in the Western Balkans in line with European perspective - InMuSWB" (G. Lechleitner / Projektmanagement, C. FenneszJuhasz, N. Wallaszkovits und J. Spitzbart; EACEA-TEMPUS IV, Joint Projects; 517098-TEMPUS-1-2011-1-RS-TEMPUS-JPCR; Okt. 2011-Okt. 2014) standen die Teilnahme am Forum von Musikuniversitäten (in Novi Sad, Gerda Lechleitner) sowie ein teacher's training (in Wien: Tamara Karača-Beljak) und Kurse in Digitalisierung und Archivierung (in Sarajevo, Nadja Wallaszkovits und Johannes Spitzbart) auf dem Programm.

Das im Dezember 2012 mit Unterstützung der Fundação Calouste Gulbenkian (Portugal) begonnene Projekt „Recuperação e tratamento documental de uma coleção de discos de 78rpm de música portuguesa doados à Universidade de Aveiro" (Kooperation mit dem Department für Ethnomusikologie der Universidade Aveiro, Portugal) wurde mit einem zweiwöchigen Workshop in Wien weitergeführt.

Mit Ludwig Maximilian Breuer (Institut für Germanistik, Universität Wien) wurde eine Kooperation zur Erstellung von Transkripten zu Wiener Dialektaufnahmen aus den Beständen des Phonogrammarchivs begonnen.

Christiane Fennesz-Juhasz verfasste ein Arbeitsprogramm zu einer ersten Kooperation des Phonogrammarchivs mit den ESFRI-Projekten CLARIN und DARIAH (Verfügbarmachen von Metadaten, kontrollierte Vokabularien und ausgewählte Bestände).

Im Rahmen des CD-Projekts „Klingende Forschung - Konzertdokumentation" ist - in Kooperation mit der Kommission für Musikforschung der 
ÖAW - die Nummer 4 Schuberts späte Werke für Klavier zu vier Händen, ein Gesprächskonzert von Walburga Litschauer mit dem Duo Egri \& Pertis, erschienen (G. Lechleitner, J. Spitzbart, N. Wallaszkovits).

Für den von Erika Sieder und Walter Deutsch (ÖVLW) herausgegebenen Band Der Wechsel - Musik einer Landschaft: Totenbrauch und Leichhüatlieder im Wechselgebiet hat das Phonogrammarchiv für die CD-Beilage aus seinen Beständen Aufnahmen von Franz Schunko bereitgestellt und das Mastering der CDs übernommen (N. Wallaszkovits, K. Thenius-Wilscher).

\section{Publikationen / Vorträge}

\section{a) Audio-Editionen}

Kowar, Helmut. 2013. Musik um 1600: Die Automaten in der Kunstkammer des Kunsthistorischen Museums. (Mechanische Musikinstrumente, $8=$ OEAW PHA CD 34). Wien: VÖAW.

In Kooperation mit dem Phonogrammarchiv entstandene CD-Publikation: Litschauer, Walburga. 2013. Schuberts späte Werke für Klavier zu vier Händen: "Kämpfe der Leidenschaften und des Verstandes". (Klingende Forschung, 4 = OEAW PHA CD 33). Wien: VÖAW. [Aufnahme: J. Spitzbart und N. Wallaszkovits; Mastering: N. Wallaszkovits; Redaktion: G. Lechleitner]

\section{b) Video-Editionen}

Schöpf, Jürgen (ed.). 2013. European Acoustic Heritage. Wien: Phonogrammarchiv. [Doppel-DVD; technische Realisierung: Johannes Spitzbart]

Feldforschungsaufnahmen (DVD-Publikationen) der RMB-Collection (Orbis Musicarum = OM). Göttingen: Cuvillier:

Brandl, Rudolf M. 2013. Kunqu in Wien. (OM 200A-C).

Brandl, Rudolf M. \& Bernhard Graf. 2013. Wiener Volksliedwerk - „Die Musik der Brüder Schrammel“: Matinée zum 90. Geburtstag von Walter Deutsch am 10.6.2013, mit dem Thalia-Quartett. (OM 198); Ostern in Olympos/Karpathos 2013: Liturgien \& Bräuche, Musik. (OM 199A-I); Wiener Lieder 2003 mit Walter Hojsa \& Kurt Schaffer und 2013 mit Rudi Koschelu und Herbert Bäuml beim Hernalser Bezirksfest. (OM 201); „Ja wos waß denn die Wöld, wos a Weana-Herz fühlt ..." im Kultur-Café "Max" am 2.9.2013 mit dem Kurt Girk Trio. (OM 202); „Weanaliad, goldenes, brauchst di net kränken ..." am 5.9.2013 im Weinlokal "Zum G'spritzten" mit dem Kurt Girk Trio. (OM 203); Musikantenstammtisch am 17.9.2013 im Heurigen Hengl-Haselbrunner mit Rudi Koschelu und Herbert Bäuml. (OM 204); „Ihr werd's no wana um die Schrammeln" - Musikalischer Abend des Vereins "Wiener Volkskunst" am 18.9.2013 mit Leopold Großmann, Elisabeth Reichert, Margarita Touschek, Peter Rosen, Malat-Trio. (OM 205); "Dos is dos Weana Bluat, das in uns wurln tuat" am 24.9.2013 im Heurigen Hengl-Haselbrunner mit Tini Kainrath und Tommy Hojsa, Helmut Emersberger, Karl Zacek, Kurt Girk. (OM 206); Robert Reinagl \& Walter Czipke sind „Die Mondscheinbrüder", im Heurigen Hengl-Haselbrunner am 8.10.2013. (OM 207); „Erst waunns aus wird sein mit aner Musi und an Wein": Rudi Koschelu und Herbert Bäuml beim Musikantenstammtisch am 15.10.2013 im Heurigen Hengl-Haselbrunner. (OM 208); „Wiener Dudler und harbe Danz" am 12.11.2013 im Heurigen Hengl-Haselbrunner mit Tini Kainrath, "Weana Spatz'n", Helmut Emersberger, Karl Zacek. (OM 209); „Drahren tan ma, Drahrer san ma“: Rudi Koschelu und Herbert Bäuml beim Musikantenstammtisch am 19.11.2013 im Heurigen Hengl-Haselbrunner. (OM 210); "Weana Spatz'n-Club", Tini Kainrath - Tommy Hojsa und Gäste am 4.12.2013 im Heurigen „Herrgott aus Sta“. (OM 211). 


\section{c) Schriftliche Publikationen}

\section{Selbstständige bzw. herausgegebene selbstständige Publikationen}

Fennesz-Juhasz, Christiane \& Emmerich Gärtner-Horvath (Hg., Zusammenstellung und Kommentar). 2013. Dschila le Romendar andar o Burgenland / Lieder der burgenländischen Roma. Uprutni Schica / Oberschützen: Burgenländisches Volksliedwerk.

Studemund-Halévy, Michael, Christian Liebl \& Ivana Vučina Simović (eds.). 2013. Sefarad an der Donau: Lengua y literatura de los sefardies en tierras de los Habsburgo. Barcelona: Tirocinio.

\section{Beiträge in Fachzeitschriften und Sammelwerken}

Huber, Christian. 2013. "Subject and object agreement in Shumcho". In: Owen-Smith, Thomas \& Nathan W. Hill (eds.). Trans-Himalayan Linguistics. (Trends in Linguistics, Studies and Monographs, 266). Berlin \& Boston: de Gruyter, 221-274.

Kowar, Helmut. 2013. "The Music of the Walbaum Cabinet ca. 1620-25". Mechanical Music (Journal of the Musical Box Society International) 59 (3): 8-15.

Kowar, Helmut. 2013. „,Wir machen Musik!' Trompeterautomat, Schiff und Bacchuswagen des Hans Schlotheim“. Jahrbuch des Kunsthistorischen Museums Wien 13/14 (2011/2012): 119-159.

Kowar, Helmut. 2013. „Die Revolution im Wohnzimmer“. In: Boisits, Barbara (Hg.). Musik und Revolution: Die Produktion von Identität und Raum durch Musik in Zentraleuropa 1848/49. Wien: Hollitzer, 433-456.

Lechleitner, Gerda. 2013. „Ideen, Strategien, Ansprüche - und was bleibt?“ biblos. Beiträge zu Buch, Bibliothek und Schrift 62 (1): 81-97.

Lechleitner, Gerda. 2013. "We never collect 'a song', but only a version of it: Reflections on Early Folk Music Recordings". In: Procházková, Jarmila (ed.). As Recorded by the Phonograph. Brno: Etnologický ústav AV CR, v.v.i., Praha, 43-56.

Lechleitner, Gerda. 2013. “Can Archives Contribute to Established Traditions? On the Interaction Between Archives and Tradition". In: Richter, Pal (ed.). Musical Traditions Discovery, Inquiry, Interpretation, and Application: XXVI European Seminar in Ethnomusicology. Budapest: MTA, 365-376.

Lechleitner, Gerda \& Nona Lomidze. 2013. "Production and Process: Creative Acting and Perceiving. A Case Study on Georgian Multipart Singing". In: Ahmedaja, Ardian (ed.). Local and Global Understandings of Creativities: Multipart Music Making and the Construction of Ideas, Contexts and Contents. Newcastle, UK: Cambridge Scholars Publishing, 77-88.

Liebl, Christian. 2013. „Sefarad im Phonogrammarchiv: Cappon, Cantors and Canetti“. In: StudemundHalévy, Michael, Christian Liebl \& Ivana Vučina Simović (eds.). Sefarad an der Donau: Lengua y literatura de los sefardies en tierras de los Habsburgo. Barcelona: Tirocinio, 371-384.

Schüller, Dietrich. 2013. "Introduction to IASA-TC 05: Handling and Storage of Audio and Video Carriers". In: Lin, S.C. (ed.). Convergence, Collaboration and Creativity: Essays from the 2012 Taiwan e-Learning and Digital Archives Program International Conference. (International Journal of Humanities and Arts Computing, 7). Edinburgh: Edinburgh University Press, 216-221.

Schüller, Dietrich. 2013. „20 Jahre Memory of the World Programm der UNESCO“. biblos. Beiträge zu Buch, Bibliothek und Schrift 62 (1): 121-127.

Wallaszkovits, Nadja. 2013. "Digitisation of Small Sound Collections: Problems and Solutions". In: Duranti, L. \& E. Shaffer (eds.). The Memory of the World in the Digital Age: Digitization and Preservation. An international conference on permanent access to digital documentary heritage. UNESCO: Vancouver, Canada, 1440-1450. [Online: <http://www.ciscra.org/docs/UNESCO_MOW2012_Proceedings_FINAL_ ENG_Compressed.pdf>] 
Wallaszkovits, Nadja \& Heinrich Pichler. 2013. „Ausgewählte Probleme mit Rauschunterdrückungsverfahren bei der Archivierung analoger Consumer-Tonbandformate". In: Bildungswerk des Verbandes Deutscher Tonmeister Leipzig (Hg.). Proceedings der 27. Tonmeistertagung. Köln: VDT International Convention, 338-356.

\section{Online-Publikationen}

Huber, Christian. 2013. „Das Phonogrammarchiv der ÖAW - Aspekte der Archivierung audiovisueller Daten“. Online: <http://homepage.univie.ac.at/ludwig.maximilian.breuer/Ch.\%20Huber\%20-\%20 Das\%20PhA\%20-\%20Aspekte\%20Archivierung\%20AV-Daten\%20(2013-05-24).pdf>.

Schüller, Dietrich. 2012 [online 2013]. "The Role of Digitization in the Preservation of Audiovisual Documents." In: Preservation of Digital Information in the Information Society. Proceedings of the International Conference, Moscow, 3-5 October, 2011. Moskau 2012, S. 248-256. [Online: <http://www.ifapcom.ru/files/News/Images/2013/dig_pres_is_eng.pdf>]

\section{Populärwissenschaftliche Beiträge in Zeitungen etc.}

Fennesz-Juhasz, Christiane. 2013. „Liedgeschichte/n - Dschilengere historiji.“ dROMa. Romani Politika I Kultura / Tschib 38: 8-16. [Online: <http://www.roma-service.at/download/dROMa38.pdf>]

\section{Vorträge}

Brandl, Rudolf M. (08.05.2013): Langzeitforschung in Karpathos über vier Generationen. (IV. Internationale Konferenz für karpathische Volkskunde). Pigadia, Karpathos, Griechenland.

Fennesz-Juhasz, Christiane \& Gerda Lechleitner (18.01.2013): Das Phonogrammarchiv der Österreichischen Akademie der Wissenschaften. (Archive lesen. Workshop im Rahmen der UNESCO-Gastprofessur „650 Jahre Sprach- und Textkulturen“). Wien: Institut für Germanistik, Universität Wien.

Fennesz-Juhasz, Christiane (15.08.2013): Performing and Celebrating "Romanipe". (isaScience - International Summer Academy of the mdw [Universität für Musik und darstellende Kunst Wien], Panel: "The Other from Within": Romani Music. The Scandalous Representation of the Romani People and their Music). Reichenau an der Rax.

Huber, Christian (20.03.2013): Transitivitätsalternationen, Verbalkongruenz und Kasusmorphologie im Shumcho. Bern: Institut für Sprachwissenschaft, Universität Bern.

Huber, Christian (24.05.2013): Das Phonogrammarchiv der ÖAW - Aspekte der Archivierung audiovisueller Daten. (Gastvorlesung im Rahmen des PS Sprachwissenschaft 100141 Diachrone und synchrone Aspekte der Stadtsprachenforschung). Wien: Institut für Germanistik, Universität Wien.

Huber, Christian (14.11.2013): Methodik in der Feldforschung. (Gastvorlesung im Rahmen des PS Sprachwissenschaft 100070 Syntax in the City). Wien: Institut für Germanistik, Universität Wien.

Kowar, Helmut (07.05.2013): „Blicke zurück in die tatsächlich gelebte Musik“: ein Orgelwerk des frühen 17. Jahrhunderts. (Vortragsveranstaltung im Berufungsverfahren zur Besetzung der Leitungsfunktion des Phono-grammarchivs). Wien: ÖAW.

Kowar, Helmut (24.06.2013): Musik um 1600. Die Automaten in der Kunstkammer des Kunsthistorischen Museums. (Vortrag zur CD Präsentation). Wien: ÖAW.

Kowar, Helmut (12.11.2013): Renaissancemusik live. Die Automaten der Kunstkammer. (Vortrag im Rahmen der Reihe „Forschung im Museum“). Wien: Kunsthistorisches Museum.

Lechleitner, Gerda (05.09.2013): Historical voices reloaded - rethinking archival responsibilities compared to intangible heritage. (Cultural Mapping and Musical Diversity, XXIX European Seminar in Ethnomusicology). Bern: Universität Bern. 
Lechleitner, Gerda (13.09.2013): Zulu recordings from 1908: a conflict between "tradition" and "modernity". (Third Symposium of the ICTM Study Group for Multipart Music). Budapest: Institute of Musicology, Hungarian Academy of Sciences.

Lechleitner, Gerda (11.10.2013): The Phonogrammarchiv and the digital world: pioneering and current challenges. Valladolid: Universidad de Valladolid, Facultad de Filosofía y Letras.

Lechleitner, Gerda (18.10.2013): Between past and present - documentation, preservation of sound and the Phonogrammarchiv's role in the "new world" of (music) museums. (Symposium Glazba um Muzeju - Muzeji Glazbe/Music in Museums - Museums of Music). Zagreb, Kroatien.

Lechleitner, Gerda (26.10.2013): Audiovisuelles Erbe und "digital humanities" - Archive im Schnittpunkt von Bewahren und Verbreiten. (Keynote, Kolloquium Memoriav meets Humanities - die Digital Humanities und das audiovisuelle Kulturgut). Neuchâtel: Musée d'ethnographie de Neuchâtel.

Lechleitner, Gerda (28.11.2013): Statement und Teilnahme an der Podiumsdiskussion. (Authentisch im Netz? Von der Herausforderung, Wissenschaft und Kultur zu virtualisieren. Eine Veranstaltung der Österreichischen Mediathek in Kooperation mit maa - Medien Archive Austria). Wien: Technisches Museum.

Liebl, Christian (30.07.2013): The Vienna Phonogrammarchiv - collections, catalogues and current strategies. (IAML-Konferenz 2013). Wien: Campus der Universität Wien.

Liebl, Christian (25.10.2013): Das Phonogrammarchiv - Österreichs wissenschaftliches audiovisuelles Archiv. (Vortrag im Rahmen der Lehrveranstaltung 080016 VO-L B230 Spezielle Felder: Visuelle und Materielle Kultur - WS 2013; Ass.-Prof. Mag. Dr. Bernhard Fuchs, Institut für Europäische Ethnologie, Universität Wien). Wien: Phonogrammarchiv.

Remmer, Ulla (22.11.2013): Vedisches aus dem Phonogrammarchiv: Brahmanische Techniken zur Memorisierung der Texte. (40. Österreichische Linguistiktagung, Workshop „Historisch-vergleichende Sprachwissenschaft"). Salzburg: Universität Salzburg.

Schabus, Wilfried (27.09.2013): Die „Kärntner“ Hutterer in Kanada. (Vortrag mit Bildpräsentation und Tonbeispielen). Arnoldstein: Kulturhaus Arnoldstein.

Schöpf, Jürgen (21.01.2013): Restudying Urban Soundscapes in Vienna, 1980 and 2012. (Patrimoine Sonore Européen - Forschungszentrum für Raumklang und urbane Umwelt, Hochschule für Architektur). Grenoble, Frankreich. [Online: <http://www.cresson.archi.fr/ECHO/EAHgrenoble/EAHgrenoble.html>]

Schöpf, Jürgen (19.04.2013): Restudying Urban Soundscapes in Vienna, 1980 and 2012. (Mesa Redonda, AGADIC). Pontevedra, Spanien.

Schöpf, Jürgen (22.05.2013): Sprache und Musik: Alte Fragen - neue Antworten? Graz: Institut für Ethnomusikologie der Universität für Musik und darstellende Kunst.

Schöpf, Jürgen (01.06.2013): Ethnomusicology, soundscapes, and soundscape art. ("Music \& Space", Joint Meeting of the Austrian National Committee in the ICTM together with the National Committees of Italy and Switzerland). Mals/Malles Venosta, Italien: Kulturhaus Burgeis.

Schöpf, Jürgen (07.06.2013): The interdisciplinary potential of language documentation. (Language Documentation: Past - Present - Future). Hannover: VolkswagenStiftung. [Online: <http://www. volkswagenstiftung.de/dobes-conference.html>]

Schöpf, Jürgen (01.07.2013): Zum Verhältnis von Sprache und Gesang in sogenannten „Tonsprachen“. München: Hochschule für Musik und Theater.

Schöpf, Jürgen (01.07.2013): Seminar Pfeifenensembles aus Botsuana und Litauen. München: Hochschule für Musik und Theater.

Schüller, Dietrich (27.02.2013): Audiovisual preservation. (Panel IFAP Special Event Information and Knowledge for All: Emerging Trends and Challenges). Paris: UNESCO.

Schüller, Dietrich (11.09.2013): Internet and its influence on quality and authenticity of audiovisual 
documents. (International Conference Internet and Socio-Cultural Transformations in the Information Society, 8.-11.9.2013). Sachalin, Russische Föderation. [Online: <http://ifapcom.ru/files/News/ Images/2013/sakhalin/presentations/schueller.pdf>]

Wallaszkovits, Nadja (23.04.2013): Digitisation of Research Sound Collections. Sarajevo: Department of Musicology and Ethnomusicology, Academy of Music, University of Sarajevo.

Wallaszkovits, Nadja (07.10.2013): Spare Parts and Equipment Availability in Audiovisual Archiving. (IASA Annual Conference). Vilnius.

Wallaszkovits, Nadja (20.10.2013): A Contribution to the History of Field Tape Recording, 19391940. (135th AES Convention New York 2013). New York. [Online: <http://www.aes.org/events/135/ historical/?ID=3823>] 